\title{
Geographic Variation in Statin Use for Complex Acute Myocardial Infarction
} Patients: Evidence of Effective Care?

John M. Brooks, PhD*

University of lowa, College of Pharmacy and College of Public Health

115 S. Grand, S515 PHAR, lowa City, IA 52242

Phone: (319) 335-8763

Fax: (319) 353-5646

john-brooks@uiowa.edu

Elizabeth Cook, M.S.

University of lowa, College of Pharmacy

115 S. Grand, S553 PHAR

lowa City, IA 52242

Phone: (319) 384-2815

Fax: (319) 353-5646

elizabeth-cook@uiowa.edu

Cole Chapman, B.S.

University of lowa, College of Pharmacy

115 S. Grand, S553 PHAR, lowa City, IA 52242

cole-chapman@uiowa.edu

Puttarin Kulchaitanaroaj , M.S.

University of lowa, College of Pharmacy

115 S. Grand, S553 PHAR

lowa City, IA 52242

puttarin@gmail.com

Elizabeth Chrischilles, M.S., PhD

University of lowa College of Public Health

105 River St., S424 CPHB, lowa City, IA 52242

Phone: (319) 384-1575

e-chrischilles@uiowa.edu

Stephen Welch, PhD

University of lowa, College of Pharmacy

115 S. Grand, S553 PHAR, lowa City, IA 52242

stephen-welch@uiowa.edu

Jennifer Robinson, M.D., M.P.H.

University of lowa College of Public Health,

105 River St., S455 CPHB, lowa City, IA 52242

Phone: (319) 384-1563

jennifer-robinson@uiowa.edu

${ }^{*}$ Corresponding Author 
Funding Source: This project was supported by an Agency for Healthcare Research and Quality grant (1R21HS019574-01) under the American Recovery and Reinvestment Act of 2009.

Manuscript Word Count: 3,348

Number of Text Pages: 20

Number of Figures: 2

Number of Tables: 3

Brief Title: Variation in Statin Use by Patient Complexity 


\section{Abstract}

Background: Despite strong evidence to designate statin use for secondary prevention of cardiovascular disease as "effective care" observational studies show that many patients with cardiovascular disease do not receive statins. This suggests that statin prescribing decisions for complex cardiovascular disease patients are preference sensitive.

Objectives: To evaluate local area variation in statin prescribing for subsets of complex patients after acute myocardial infarction to assess whether current statin prescribing patterns fit profiles of either "effective care" or "preference-sensitive care"

Research Design/Subjects: Retrospective cohort study of 124,618 Medicare patients with fee-for service Parts A, B, and D benefits who were hospitalized with AMI in 2008 or 2009 with no evidence of $\mathrm{AMI}$ in the past 12 months.

Measures: Patient complexity was defined by the presence of diabetes, heart failure, and chronic kidney disease in the year before AMI admission. Local area practice styles for "no statin", "lower-intensity statins", and "high-intensity statins" were measured using the Driving Area for Clinical Care method. Statin prescribing rates for complex patient subsets were contrasted across patients grouped by local areas practice styles.

Results: Lower statin treatment rates are observed for patients with complex conditions, especially among those with heart failure. However, substantial local area variation in statin prescribing is observed across all complex patient groups. 
This is a final peer-reviewed manuscript.

For a published version, please go to http://journals.Iww.com/lww-

medicalcare/Abstract/2014/03001/Geographic_Variation_in_Statin_Use_for_Complex.9.aspx.

Conclusion: Despite guidelines promoting the use of statins for secondary prevention

for CVD patients, substantial local area variation suggests that patient and provider beliefs and preferences weigh heavily in statin prescribing decisions. 


\section{Introduction}

\section{Given the strength of evidence from numerous randomized controlled trials} (RCTs), statin use for the secondary prevention of future cardiovascular events for patients with cardiovascular disease (CVD) has been designated as "effective care" ${ }^{1,2}$ Effective care is defined by the Dartmouth Atlas as "services of proven effectiveness that involve no significant tradeoffs - all patients with specific medical needs should receive them." ${ }^{3,4}$ Effective care is characterized by strong evidence that provides little clinical discretion so that non-medical factors should have little influence on treatment choice. ${ }^{4,5}$ Clinical guidelines also appear to support the effective care categorization of statin use for patients with CVD. ${ }^{6,7}$ In fact, it is thought that most patients will need a high-intensity statin to achieve their cholesterol goals. ${ }^{8-15}$

However, it is not clear whether the designation of statins as effective care for all CVD patients reflects the practice beliefs of providers. CVD patients discharged from hospitals that promoted guideline care had statin discharge prescribing rates ranging from 77 to $90 \% .{ }^{16-18}$ Only $54 \%$ of a sample of Medicare beneficiaries filled a statin prescription within the 30 days after an acute myocardial infarction (AMI) discharge,$^{19}$ and only $52 \%$ of patients 65 and older in a managed care plan filled a statin prescription within 90 days after an AMI discharge. ${ }^{20}$ In addition, substantial geographic variation in statin spending per Medicare beneficiary was found. ${ }^{21}$ Lack of awareness of the clinical evidence does not appear to be a source of this apparent statin underuse as $96 \%$ of physicians identify a low density lipoprotein cholesterol (LDL-C) of less than $100 \mathrm{mg} / \mathrm{dl}$ as the treatment goal for high-risk patients. ${ }^{22}$ 
Dartmouth provides a contrasting "preference sensitive" category of medical care in which treatment decisions involve tradeoffs across outcomes. ${ }^{4,5}$ It may be that the benefits and adverse-effect risks of statins are heterogeneous across patients and that providers believe that the risks of adverse effects from statins may outweigh statin benefits for many CVD patients. RCTs provide some evidence of heterogeneous statin effects across patients. Absolute CVD benefits from statin therapy vary with patient age and are thought to vary with the presence of diabetes (more benefit), heart failure (little or no benefit), and chronic kidney disease (variable benefit). ${ }^{23-28}$ While the statin adverse-effect risks found in RCTs are considered small relative to statin benefits, ${ }^{29,30}$ it has been suggested that favorable patient selection in RCTs resulted in adverse-effect risk estimates that are lower than what occurs in practice. ${ }^{31-34}$ Statin adverse-effects have been shown to vary with statin intensity, patient age, gender, weight, health behaviors, comorbidities, and concomitant drug use. ${ }^{32,35-41}$ Given these potential tradeoffs, in its recommended approach for patient-centered care, the American Geriatrics Society Expert Panel on the Care of Older Adults with Multimorbidity used statins as an example of a "preference sensitive decision" that may "confer long-term benefits but cause short-term harm". ${ }^{42}$

Given that complex CVD patients are often underrepresented in RCTs ${ }^{43,44}$ we theorized that greater CVD patient complexity implies greater evidence uncertainty and the more that statin use is considered preference-sensitive by providers. Our objective was to assess whether local area statin prescribing patterns for complex patients discharged with acute myocardial infarction (AMI) fit profiles of either "effective care" or "preference-sensitive care". Complex AMI patients were defined here using 
This is a final peer-reviewed manuscript.

For a published version, please go to http://journals.Iww.com/lww-

medicalcare/Abstract/2014/03001/Geographic_Variation_in_Statin_Use_for_Complex.9.aspx.

combinations of conditions suggested to affect statin effectiveness: diabetes, heart failure (HF), and chronic kidney disease (CKD). ${ }^{23-28}$ We hypothesized that with evidence less certain for complex AMI patients, statin prescribing rates after AMI will be lower for complex patient and that geographic variation in statin use will increase with patient complexity. We also theorized that, with higher adverse-effect risks, prescribing rates for high-intensity statins will fall with patient complexity. This study was approved by the University of lowa Institutional Review Board.

\section{Methods}

Data and Sample

All Medicare claims files, enrollment information, and Part D prescription drug events were obtained from the Chronic Condition Data Warehouse (CCW www.ccwdata.org) for patients hospitalized with an AMI in 2008 and 2009 using the CCW definition of AMI (an inpatient stay with the primary diagnosis code $410 . x 1$ at any time during the year). The acute hospital admission date for each AMI served as the index date for the AMI. The length of stay for each AMI was based on all Medicare institutional claims (acute, long term care hospital, inpatient rehabilitation facility, criticalaccess hospital, and short-term nursing facility) with overlapping admission and discharge dates following the initial acute hospital AMI admission. The institutional stay discharge date was the day the patient was discharged home. We excluded AMls if the patient (1) did not survive the AMI institutional stay; (2) had an AMI within 12 months prior to the index date; (3) was less than 66 years old at the index date to ensure at least one year of Medicare eligibility prior to the index date; (4) did not have continuous 
Medicare Parts A and B enrollment during the 12 months prior to the index date; (5) was not continuously enrolled in Medicare Part D during the 6 months to the index date; and (6) did not have continuous Medicare Parts A, B and D enrollment during period from the discharge date to the minimum of the patient death month or 12 months after discharge. To ensure a consistent statin measurement period after discharge we further excluded patients who used hospice or skilled nursing care; were readmitted to inpatient care; or died during the 30 days after the institutional stay discharge date. ${ }^{45}$ Finally, because we use driving times between ZIP codes to define local areas driving times have inconsistent meaning for geographically non-contiguous areas (e.g. islands not connected by bridges), we restricted our sample to patients living in the continental U.S. at AMI admission. The final cohort was 124,813 patients.

\section{Patient Complexity}

We define AMI patient complexity using combinations of diabetes, chronic kidney disease (CKD), and heart failure (HF) diagnosed before the index AMI. Earlier studies suggested these conditions are associated with statin effectiveness. ${ }^{23-28} \mathrm{We}$ modified the validated Chronic Condition Data Warehouse (CCW) definitions of these conditions to accommodate our 1-year look back period rather than the 2year period specified by CCW. The diagnosis codes used to identify each condition can be found in the online, supplemental digital content Appendix. To identify CKD we searched for at least one Medicare inpatient, skilled nursing facility or home health claim or two hospital outpatient or physician claims with the relevant diagnosis codes in any position on the claims. To identify HF and diabetes, we searched for at least one inpatient, hospital outpatient or physician claim with relevant diagnosis codes in any 
position on the claim. Patients were then stratified into eight complex combinations given these diagnoses before AMI index - (1) no prior HF, CKD or diabetes; (2) HF only; (3) CKD only; (4) diabetes only; (5) HF and CKD only; (6) HF and diabetes only; (7) CKD and diabetes only; (8) All three prior conditions.

\section{Measures of Statin Intensity Prescribing Intent}

Our measurement goal was to assess prescribing intent by statin intensity for each patient at AMI discharge. High-intensity statins were defined as those that can lower LDL-C by $50 \%$ or more: atorvastatin $40,80 \mathrm{mg}$; and rosuvastatin $20,40 \mathrm{mg}$. Lowerintensity statins were defined as those that lower LDL-C less than $50 \%$ : atorvastatin 10 , 20mg; fluvastatin 20,40,80mg; lovastatin 10,20,40,80mg; rosuvastatin 10; pravastatin 10,20,40,80mg; rosuvastatin 5mg; simvastatin 5, 10,20,40,80mg. ${ }^{10}$ To measure prescribing intent we used (1) Part D claims during 30 days after the AMI discharge date; and (2) estimates of statins available to the patient at home at AMI discharge based on previous prescription dates and days supplied on Part D claims. Two binary treatment variables (lower and high) were specified for each patient. If a patient's first statin prescription after discharge was a high-intensity statin or if a patient filled two or more lower-intensity statin prescriptions of the same drug within 2 days of the first statin prescription with doses summing to high (e.g. two atorvastatin $20 \mathrm{mg}$ prescriptions), the patient was assigned lower $=0$ and $\mathrm{high}=1$. All other statin prescription combinations during the 30 days after AMI discharge resulted in lower $=1$ and high $=0$. It was also possible that a patient was prescribed a statin on AMI discharge but had sufficient statins at home to cover the first 30 days after 
This is a final peer-reviewed manuscript.

For a published version, please go to http://journals.Iww.com/lww-

medicalcare/Abstract/2014/03001/Geographic_Variation_in_Statin_Use_for_Complex.9.aspx.

AMI discharge. To account for this, if a patient had no statin prescriptions in the 30 days after discharge and had at least 30-days of a high-intensity statin at home, the patient was assigned lower $=0$ and high $=1$. Likewise, if a patient had no statin prescriptions in the 30 days after discharge and had at least 30 days of a lower-intensity statin at home at the patient was assigned lower $=1$ and high $=0$. All other patients were assigned as "no statin" or lower $=0$ and high $=0$.

\section{Local Area Practice Style Measures of Statin Intensity}

We measured local area statin practice style as the average intent of physicians in the local area around each patient resident ZIP code to prescribe statins by intensity at AMI discharge. Because discharge prescribing intent is less clear for patients with statins available at home on discharge, we used only the patients with no statins at home on their AMI discharge date $(\mathrm{N}=79,285)$ in our measures. Practice styles were measured at the patient ZIP code-level using the driving area for clinical care (DACC) method. ${ }^{46}$ The DACC method creates "local areas" around each patient residence ZIP code by consecutively adding patients from the next closest ZIP codes based on driving times between zip codes until a threshold number of patients have been reached. ${ }^{46}$ Local area practice style measures based on the DACC method have explained a larger portion of treatment variation than other local area definitions and effectively balanced

measured covariates. ${ }^{46-48}$ We used a local area size threshold of 100 patients. For the patients in the local areas around each ZIP code using the DACC method, area treatment ratios (ATR) for "no statin", "lower-intensity statins" and "high-intensity statins" were estimated. Each ATR was calculated as the ratio of the number of patients in the local area around a ZIP code that received the respective statin intensity after AMI over 
the sum across these patients of their predicted probabilities of receiving that statin intensity after AMI. Probabilities were assigned to each patient of receiving no statins, a lower-intensity statin, and a high-intensity statin based their baseline covariates using a multinomial model of statin intensity choice. The multinomial model specified measures for patient demographics; baseline comobidities for both the year prior to the AMI admission and during the index AMI stay including conditions described as statin side effects (myopathy, rhabdomyolysis, renal events, and hepatic events); medications used during the 180 days prior to the AMI admission; AMI diagnosis-type on admission; procedures during the AMI stay; complications during the AMI stay; the number days of the AMI institutional length of stay spent in intensive care and critical care; other medications filled immediately post discharge (beta blockers, renin-angiotensin system antagonists); Part D variables including premium levels, benefit phase at AMI index date and beneficiary accumulated total and out-of-pocket drug costs prior to AMI index; whether patients were Medicaid dual-eligible in their AMI index month; patient low-income status, and socioeconomic characteristics for each patient residence zip code (per capita income, poverty rate, education level, English speaking percentage, rural/urban residence, life expectancy). Full definitions of these variables are included in the online, supplemental digital content Appendix. A ZIP code with an ATR greater than 1 for a specific statin intensity had a local area practice style in which that statin intensity was used at a rate higher than average given the baseline characteristics of the patients in the local area. A ZIP code with an ATR less than 1 had a local area practice style in which the respective statin intensity was used less than average. 


\section{Analysis}

Patients in our full sample $(\mathrm{N}=\mathbf{1 2 4 , 8 1 3 )}$ were assigned the ATR values for no-statin, lower-intensity statins, and high-intensity statins based on their residence ZIP code. We then stratified our sample by patient complexity based on combinations of prior CKD, HF and diabetes. For each complex patient combination we estimated treatment rates by statin intensity. Patients were grouped based on the quintiles across the full sample of each statin-intensity specific ATR. We then estimated treatment rates by statin intensity for each complex patient combination across ATR quintiles and report the range in variation in statin treatment rates across quintiles by statin intensity.

\section{Results}

Table 1 contains the characteristics of our sample by available statin-intensity after AMI discharge. Statins were not available to $38 \%$ of patients in our sample, a lower-intensity statin was available to $50 \%$, and a high-intensity statin was available to $12 \%$. Patients with a statin available after discharge tended to be younger; had fewer comobidities (lower Charlson score); were more likely free of the 3 complex conditions (heart failure, CKD, diabetes); had fewer conditions before AMI or during their AMI stay that are considered statin adverse-effects; appeared to have more severe AMls as indicated by a higher percentage of patients having an anterior wall AMI, a lower percentage having a non-ST elevation AMI, and higher percentage having cardiac 
Code. In addition, patients with a history of statin use were more likely to have statins available after discharge.

Table 2 shows the distribution of patient characteristics after grouping patients by the high-intensity statin area treatment ratio (ATR) associated with their residence ZIP code. The percentage of patients that had a high-intensity statin available after AMI discharge varied from $6 \%$ to $20 \%$ across the quintiles. The ZIP code with the highest high-intensity ATR had a high-intensity statin treatment rate of $33 \%$ whereas the local areas around 73 ZIP codes had high-intensity statin treatment rates of zero. Trends in the measured covariates remained across the patients grouped by quintiles of the high-intensity statin ATR, but these differences are small relative to the covariate differences when patients were grouped by available statin intensity in Table $1 .^{49}$ Similar findings of smaller covariate variation were observed when patients were grouped by the "no statin" and lowintensity statin ATRs (not shown). "No statin" treatment rates ranged from $21 \%$ to $69 \%$ across the ZIP codes with the minimum and maximum "no statin" ATRs, respectively, and low-intensity statin treatment rates ranged from $15 \%$ to $61 \%$ across ZIP codes with the minimum and maximum low-intensity statin ATRs, respectively. Figures 1 and 2 contain maps of the northeastern portion of the United States showing the quintile groups of the high-intensity ATR and no-statin ATRs, respectively. These maps illustrate substantial with-region variation in local area statin practice styles.

Average "no statin" treatment rates in Figure 2 were $32 \%$ in the white areas ( $1^{\text {st }}$ quintile) and $44 \%$ in the dark green areas $\left(5^{\text {th }}\right.$ quintile). 
Table 3 shows the percentages of patients with statins available after AMI discharge for the full sample, the sample stratified by whether patients had each prior complex condition (CKD, heart failure, and diabetes); and the sample stratified into complex combinations. Table 3 also shows the range in treatment rates between the first and fifth quintiles by statin intensity for each respective ATR-based local area practice measure. While $61.9 \%$ of our sample had a statin available after AMI discharge, rates were lower for patients with prior complex conditions. Nearly $70 \%$ of patients without heart failure, diabetes, or CKD before AMI had a statin available after discharge, whereas, only $56.6 \%$ of patients with heart failure, $57.2 \%$ of patients with CKD, $61.5 \%$ of patients with diabetes had a statin available after discharge. Comparing rates across complex combinations shows lower statin rates occur mainly for patients with prior heart failure or CKD. Specifically, patients with both prior heart failure and CKD had the lowest percentage of statin availability after AMI discharge (52.6\%), followed by patients with HF only (56.5\%), and patients with all 3 prior conditions (56.5\%). Patients with only diabetes before AMI had statin availability rates similar to patients with no prior conditions (68.5\%). Patients with heart failure and CKD also had the lowest high-intensity statin treatment rate (9.3\%) and patients with no prior conditions and patients with only prior diabetes had the highest high-intensity statin treatment rates ( $14.1 \%$ and $14.0 \%$, respectively).

Substantial geographic variation in statin availability existed across all complex combinations after AMI discharge, but the extent of geographic variation was not consistent across the complex combinations. For both the low-intensity statin and highintensity statin ATRs, the largest rate difference across quintiles was for patients with no 
This is a final peer-reviewed manuscript.

For a published version, please go to http://journals.Iww.com/lww-

medicalcare/Abstract/2014/03001/Geographic_Variation_in_Statin_Use_for_Complex.9.aspx.

prior heart failure, CKD, or diabetes (18 percentage points). Geographic variation in statin use was lowest in more complex patient groups. For example, patients with all 3 prior complex conditions had the lowest rate difference in lower-intensity statins across local area quintiles (11 percentage points) and the second lowest rate difference in high-intensity statins across quintiles (11 percentage points). Patients with prior HF and CKD only had lowest had the lowest rate difference in high-intensity statins across quintiles (10 percentage points).

\section{Discussion}

Our objective was to assess whether local area statin prescribing patterns for complex patients discharged with acute myocardial infarction (AMI) fit profiles of either "effective care" or "preference-sensitive care". 4,5 Close to $62 \%$ of the Medicare patients in our sample had a statin available during the 30-days after discharge for AMI. This percentage ranged from $69.8 \%$ for patients without heart failure, diabetes and CKD, to little more than half $(52.6 \%)$ for patients with previous heart failure and CKD. Given that most providers are aware of the cholesterol reduction goals for high-risk CVD patients, ${ }^{22}$ these rates suggest that both perceived benefits and risks associated with statins are being incorporated into prescribing decisions. Our finding of lower statin rates for more complex patients supports this idea as statin adverse-effect risks have been shown to increase with patient complexity. ${ }^{32,35-41}$ It is noted that prior diabetes had little effect on statin rates is consistent with studies suggesting that statin benefits are enhanced for diabetic patients. ${ }^{23-25}$ In addition, substantial geographic variation in 
statin availability after AMI was found across the entire sample and within each complex combination. These results suggest that differences exist across local areas in either the beliefs on relationships between statins and outcomes or in the preferences that providers and patients have over the outcomes associated with statin use. Interestingly, the extent of geographic variation in statin use was lower for more complex patients. There appears to be more agreement across local areas in the lower statin treatment rates for more complex patients than the higher statin treatment rates for the less complex patients.

The ability to make inferences on variation in provider beliefs in this study is limited by the inability of our measures to differentiate between physician and patient choices. The measures used here reflect both physician prescribing behavior and the willingness of patients to fill statin the prescriptions they received. Our statin use measures understate the prescribing intent of physicians to the extent that prescriptions are unfilled by the patient. In addition, it also is possible that the geographic variation in statin use we found could be partially attributable to geographic variation in unmeasured conditions like patient frailty.

Statin rates that diminish with patient complexity and the substantial local area variation in statin rates suggest that providers consider statins to be more "preferencesensitive care" than "effective care" for secondary prevention of cardiovascular disease. Local area variation in statin use exists across all groups of complex AMI patients. However, our results do not say whether current statin utilization rates represent a correct balancing of statin benefits and risks across complex AMI patients. Further research is needed to assess whether many complex AMI patients in areas with low 
This is a final peer-reviewed manuscript.

For a published version, please go to http://journals.Iww.com/lww-

medicalcare/Abstract/2014/03001/Geographic_Variation_in_Statin_Use_for_Complex.9.aspx.

statin utilization rates are missing benefit opportunities or, in contrast, whether many

complex AMI patients in areas with high statin utilization rates and suffering adverse

side effects with little benefit gain. In context of statin use for secondary prevention of

cardiovascular disease for complex patients, this question is analogous to the question

stated many years ago by John Wennberg, "Which rate is right?". 50 
This is a final peer-reviewed manuscript.

For a published version, please go to http://journals.Iww.com/lww-medicalcare/Abstract/2014/03001/Geographic_Variation_in_Statin_Use_for_Complex.9.aspx.

\begin{tabular}{|c|c|c|c|c|c|}
\hline & \multirow[b]{2}{*}{$\begin{array}{c}\text { Total } \\
\text { Population }\end{array}$} & \multicolumn{3}{|c|}{$\begin{array}{l}\text { Intensity of Initially-Prescribed Statin } \\
\text { Availability After AMI Discharge }\end{array}$} & \multirow[b]{2}{*}{ P-value } \\
\hline & & None & Lower & High & \\
\hline $\mathrm{N}$ & 124,813 & 47,566 & 62,316 & 14,931 & \\
\hline Treatment & & & & & $<0.0001^{*}$ \\
\hline No Statin \% & 38 & 100 & 0 & 0 & \\
\hline Lower-Intensity Statin ${ }^{\mathrm{a}} \%$ & 50 & 0 & 100 & 0 & \\
\hline High-Intensity Statin ${ }^{\mathrm{a}} \%$ & 12 & 0 & 0 & 100 & \\
\hline Age & & & & & $<0.0001^{*}$ \\
\hline $66-75 \%$ & 41 & 33 & 44 & 52 & \\
\hline $76-85 \%$ & 39 & 39 & 39 & 37 & \\
\hline $86+\%$ & 21 & 28 & 17 & 12 & \\
\hline Gender & & & & & $<0.0001^{*}$ \\
\hline Male \% & 43 & 40 & 45 & 49 & \\
\hline Female \% & 57 & 60 & 55 & 51 & \\
\hline Charlson Score $^{b}$ & & & & & $<0.0001^{*}$ \\
\hline $0 \%$ & 33 & 28 & 36 & 38 & \\
\hline $1+\%$ & 67 & 72 & 64 & 62 & \\
\hline Complex Patient Combinations $^{c}$ & & & & & $<0.0001^{*}$ \\
\hline $\begin{array}{l}\text { No Prior Heart Failure, CKD or Diabetes } \\
\%\end{array}$ & 25 & 20 & 28 & 30 & \\
\hline Heart Failure only $\%$ & 16 & 19 & 15 & 14 & \\
\hline CKD only $\%$ & 5 & 5 & 5 & 5 & \\
\hline
\end{tabular}


This is a final peer-reviewed manuscript.

For a published version, please go to http://journals.Iww.com/lww-medicalcare/Abstract/2014/03001/Geographic_Variation_in_Statin_Use_for_Complex.9.aspx.

\begin{tabular}{|c|c|c|c|c|c|}
\hline Diabetes only \% & 12 & 10 & 13 & 14 & \\
\hline Heart Failure and CKD only \% & 10 & 12 & 9 & 8 & \\
\hline Heart Failure and Diabetes only \% & 12 & 13 & 12 & 11 & \\
\hline CKD and Diabetes only \% & 5 & 5 & 5 & 5 & \\
\hline Heart Failure, CKD and Diabetes \% & 15 & 17 & 14 & 13 & \\
\hline Arterial Wall $\mathrm{AMI}^{\mathrm{d}} \%$ & 6 & 4 & 7 & 9 & $<0.0001^{*}$ \\
\hline NSTEMI AMI $\%$ & 76 & 80 & 74 & 70 & $<0.0001^{\star}$ \\
\hline Catheterization During Index Stay \% & 59 & 44 & 67 & 75 & $<0.0001^{*}$ \\
\hline $\begin{array}{l}\text { Statin Rx in } 180 \text { Days Prior to Index } \\
\text { AMI \% }\end{array}$ & 47 & 26 & 60 & 59 & $<0.0001^{*}$ \\
\hline \multicolumn{6}{|l|}{$\begin{array}{l}\text { Conditions Related to Statin Side- } \\
\text { Effects }\end{array}$} \\
\hline Pre-Index AMI \% & 23 & 26 & 21 & 19 & $<0.0001^{*}$ \\
\hline During Index $\mathrm{AM}^{\dagger} \mathrm{I} \%$ & 20 & 23 & 18 & 17 & $<0.0001^{\star}$ \\
\hline Low Income Zip Code ${ }^{9} \%$ & 50 & 51 & 49 & 46 & $<0.0001^{*}$ \\
\hline
\end{tabular}

a. Based on highest statin intensity in 30 days post-index stay discharge or intensity of available 30-day supply available prior to discharge.

b. Klabunde CN et al. Development of a comorbidity index using physician claims data. Journal of Clinical Epidemiology, 200 Dec; 53(12) 1258-67.

c. HF: Heart Failure: CKD: Chronic Kidney Disease. See Appendix for CKD, HF, and diabetes ICD-9 codes

d. ICD-9 codes 410.0410 .1

e. ICD-9 $410.7 x$

f acute renal failure/acute tubular necrosis ICD-9 584.xx; acute glomerulonephritis ICD-9 580.xx. Myopathy: ICD-9-CM 728.89, 729.1, 359.4,359.8, 359.9, 710.4, 728.9, 729.8X, E942.2; CPT codes 82550, 82552, 82554, 80012, 80016, 80018, or 80019. Acute/sub-acute necrosis of liver ICD-9 570.xx; hepatitis ICD-9 573.3x; other disorders of liver ICD-9 573.8x, 573.9x.

g. Percentage of low income residents was above median in 2000 for beneficiary zip code.

h. Pearson Chi-Square statistic calculated by estimating the expected number of observations in each cell of an R-by-C table, and comparing these values with the observed number of observations in each cell of the table. The $p$-value is estimated using the ChiSquare distribution with $(\mathrm{R}-1)^{\star}(\mathrm{C}-1)$ degrees of freedom.

$* \mathrm{p}<.05$ 
This is a final peer-reviewed manuscript.

For a published version, please go to http://journals.Iww.com/Iww-medicalcare/Abstract/2014/03001/Geographic_Variation_in_Statin_Use_for_Complex.9.aspx.

\begin{tabular}{|c|c|c|c|c|c|c|c|}
\hline & \multirow[b]{2}{*}{ Total Population } & \multicolumn{5}{|c|}{$\begin{array}{c}\text { Quintile of High-Intensity Statin Area } \\
\text { Treatment Ratio } \\
\text { (Higher Area Treatment Ratio (ATR) } \rightarrow \text { ) }\end{array}$} & \multirow[b]{2}{*}{ P-value ${ }^{h}$} \\
\hline & & $1^{\text {st }}$ & $2^{\text {nd }}$ & $3^{\text {rd }}$ & $4^{\text {th }}$ & $5^{\text {th }}$ & \\
\hline $\mathrm{N}$ & 124,813 & 24,693 & 24,691 & 24,693 & 24,692 & 24,694 & \\
\hline $\begin{array}{l}\text { High-Intensity Statin Average } \\
\text { Area Treatment Ratio }\end{array}$ & 1 & 0.36 & 0.67 & 0.93 & 1.24 & 1.84 & $<0.0001^{*}$ \\
\hline \multicolumn{8}{|l|}{ Treatment } \\
\hline No Statin \% & 38 & 40 & 39 & 39 & 38 & 36 & $<0.0001^{*}$ \\
\hline Lower-Intensity Statin ${ }^{\mathrm{a}} \%$ & 50 & 54 & 52 & 50 & 48 & 45 & $<0.0001^{*}$ \\
\hline High-Intensity Statin ${ }^{\mathrm{a}} \%$ & 12 & 6 & 9 & 11 & 14 & 20 & $<0.0001^{*}$ \\
\hline \multicolumn{8}{|l|}{ 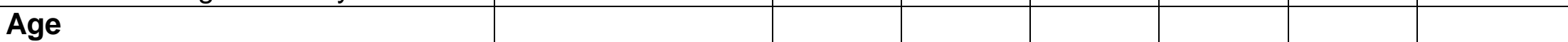 } \\
\hline $66-75 \%$ & 41 & 41 & 42 & 41 & 41 & 39 & $<0.0001^{*}$ \\
\hline $76-85 \%$ & 39 & 39 & 38 & 39 & 38 & 40 & 0.0609 \\
\hline $86+\%$ & 21 & 20 & 20 & 20 & 21 & 22 & $<0.0001^{*}$ \\
\hline \multicolumn{8}{|l|}{ Sex } \\
\hline Male \% & 43 & 44 & 44 & 43 & 43 & 42 & $<0.0001^{*}$ \\
\hline Female \% & 57 & 56 & 56 & 57 & 57 & 58 & $<0.0001^{*}$ \\
\hline \multicolumn{8}{|l|}{ Charlson Score $^{b}$} \\
\hline $0 \%$ & 33 & 36 & 34 & 33 & 33 & 31 & $<0.0001^{*}$ \\
\hline $1+\%$ & 67 & 64 & 66 & 67 & 67 & 69 & \\
\hline Complex Patient Combinations $^{c}$ & & & & & & & $<0.0001^{*}$ \\
\hline $\begin{array}{r}\text { No Prior Heart Failure, CKD or } \\
\text { Diabetes } \%\end{array}$ & 25 & 27 & 25 & 25 & 24 & 23 & \\
\hline Heart Failure only \% & 16 & 17 & 16 & 16 & 16 & 16 & 0.0775 \\
\hline CKD only \% & 5 & 5 & 5 & 5 & 5 & 5 & 0.3946 \\
\hline Diabetes only \% & 12 & 12 & 12 & 11 & 12 & 11 & 0.5881 \\
\hline Heart Failure and CKD only \% & 10 & 9 & 10 & 10 & 10 & 11 & $0.0015^{*}$ \\
\hline Heart Failure and Diabetes only \% & 12 & 11 & 12 & 12 & 12 & 13 & $<0.0001^{*}$ \\
\hline CKD and Diabetes only \% & 5 & 5 & 5 & 5 & 5 & 5 & 0.3813 \\
\hline
\end{tabular}


This is a final peer-reviewed manuscript.

For a published version, please go to http://journals.Iww.com/lww-medicalcare/Abstract/2014/03001/Geographic_Variation_in_Statin_Use_for_Complex.9.aspx.

\begin{tabular}{|c|c|c|c|c|c|c|c|}
\hline $\begin{array}{r}\text { Heart Failure, CKD and Diabetes } \\
\%\end{array}$ & 15 & 14 & 15 & 15 & 16 & 16 & $<0.0001^{*}$ \\
\hline Arterial Wall AMI ${ }^{\mathrm{d}} \%$ & 6 & 6 & 6 & 6 & 6 & 6 & $<0.0269^{*}$ \\
\hline NSTEMI AMI ${ }^{\mathrm{e}} \%$ & 76 & 74 & 75 & 76 & 76 & 78 & $<0.0001^{*}$ \\
\hline $\begin{array}{l}\text { Catheterization During Index Stay } \\
\%\end{array}$ & 59 & 62 & 60 & 60 & 58 & 56 & $<0.0001^{*}$ \\
\hline $\begin{array}{l}\text { Statin Rx in } 180 \text { Days Prior to } \\
\text { Index AMI \% }\end{array}$ & 47 & 45 & 47 & 47 & 47 & 50 & $<0.0001^{*}$ \\
\hline \multicolumn{8}{|l|}{$\begin{array}{l}\text { Conditions Related to Statin } \\
\text { Side-Effects }\end{array}$} \\
\hline Pre-Index $\mathrm{AMI}^{\dagger} \%$ & 23 & 22 & 23 & 23 & 23 & 24 & $<0.0001^{*}$ \\
\hline During Index $A M^{\dagger} I \%$ & 20 & 18 & 20 & 20 & 20 & 21 & $<0.0001^{*}$ \\
\hline Low Income Zip Code $\%$ & 50 & 52 & 50 & 51 & 50 & 46 & $<0.0001^{*}$ \\
\hline
\end{tabular}

a. Based on highest statin intensity in 30 days post-index stay discharge or intensity of available 30-day supply available prior to discharge.

b. Klabunde CN et al. Development of a comorbidity index using physician claims data. Journal of Clinical Epidemiology, 200 Dec; 53(12) 1258-67.

c. HF: Heart Failure: CKD: Chronic Kidney Disease. See Appendix for CKD, HF, and diabetes ICD-9 codes

d. ICD-9 codes 410.0410 .1

e. ICD-9 410.7x

f acute renal failure/acute tubular necrosis ICD-9 584.xx; acute glomerulonephritis ICD-9 580.xx. Myopathy: ICD-9-CM 728.89, 729.1, 359.4,359.8, 359.9, 710.4, 728.9, 729.8X, E942.2; CPT codes 82550, 82552, 82554, 80012, 80016, 80018, or 80019. Acute/sub-acute necrosis of liver ICD-9 570.xx; hepatitis ICD-9 573.3x; other disorders of liver ICD-9 573.8x, 573.9x.

g. Percentage of low income residents was above median in 2000 for beneficiary zip code.

h. Cochran-Armitage test of trend in characteristic value across patients grouped into quintiles based on local area high-intensity practice style measure. For example, the $p$ value for Age 76-85 tests whether a linear trend in the percentage of patients in this age group exists across quintiles of the high-intensity ATR-based patient groups

$* p<.05$ 
Table 3: Geographic Variation in Statin Intensity Treatment Rates for Medicare AMI Patients post-Discharge by Patient Complexity

\begin{tabular}{|c|c|c|c|c|}
\hline & $\mathbf{N}$ & $\begin{array}{c}\% \text { No Statin } \\
\left(1^{\text {st }}-5^{\text {th }} \text { quintile range }\right)^{\mathrm{a}}\end{array}$ & $\begin{array}{c}\% \text { Lower-Intensity } \\
\text { Statin } \\
\left(1^{\text {st }}-5^{\text {th }} \text { quintile range }\right)^{\text {b }}\end{array}$ & $\begin{array}{c}\% \text { High-Intensity } \\
\text { Statin } \\
\left(1^{\text {st }}-5^{\text {th }} \text { quintile range }\right)^{\mathrm{c}}\end{array}$ \\
\hline Full Sample & 124,813 & $38.1(32-44)$ & $49.9(43-57)$ & $12.0(6-20)$ \\
\hline \multicolumn{5}{|l|}{$\begin{array}{l}\text { Patients with } \\
\text { Prior Condition }\end{array}$} \\
\hline Prior HF & 66,644 & $43.4(36-49)$ & $46.1(40-53)$ & $10.5(6-17)$ \\
\hline Prior Diabetes & 54,125 & $38.6(33-44)$ & $49.5(43-55)$ & $12.0(7-19)$ \\
\hline Prior CKD & 43,690 & $42.8(36-49)$ & $46.5(41-53)$ & $10.7(6-17)$ \\
\hline \multicolumn{5}{|l|}{$\begin{array}{l}\text { Complex } \\
\text { Combinations }\end{array}$} \\
\hline No HF, CKD or D & 31,170 & $30.2(24-37)$ & $55.7(47-65)$ & $14.1(6-24)$ \\
\hline HF only & 20,451 & $43.4(35-51)$ & $46.2(39-55)$ & $10.3(5-18)$ \\
\hline CKD only & 6,597 & $37.8(33-44)$ & $50.4(43-57)$ & $11.9(6-21)$ \\
\hline D only & 14,364 & $31.4(26-38)$ & $54.5(46-62)$ & $14.0(8-23)$ \\
\hline HF and CKD only & 12,470 & $47.4(39-54)$ & $43.3(38-51)$ & $9.3(5-15)$ \\
\hline $\mathrm{HF}$ and D only & 15,138 & $40.1(34-45)$ & $48.7(43-55)$ & $11.3(7-18)$ \\
\hline CKD and D only & 6,038 & $36.4(32-43)$ & $51.2(45-58)$ & $12.4(8-20)$ \\
\hline $\mathrm{HF}, \mathrm{CKD}$, and $\mathrm{D}$ & 18,585 & $43.5(38-49)$ & $45.7(40-51)$ & $10.8(6-17)$ \\
\hline
\end{tabular}


This is a final peer-reviewed manuscript.

For a published version, please go to http://journals.Iww.com/lww-

medicalcare/Abstract/2014/03001/Geographic_Variation_in_Statin_Use_for_Complex.9.aspx.

Figure 1: Northeastern United States High-Intensity Statin Area Treatment Ratios by ZIP Code.

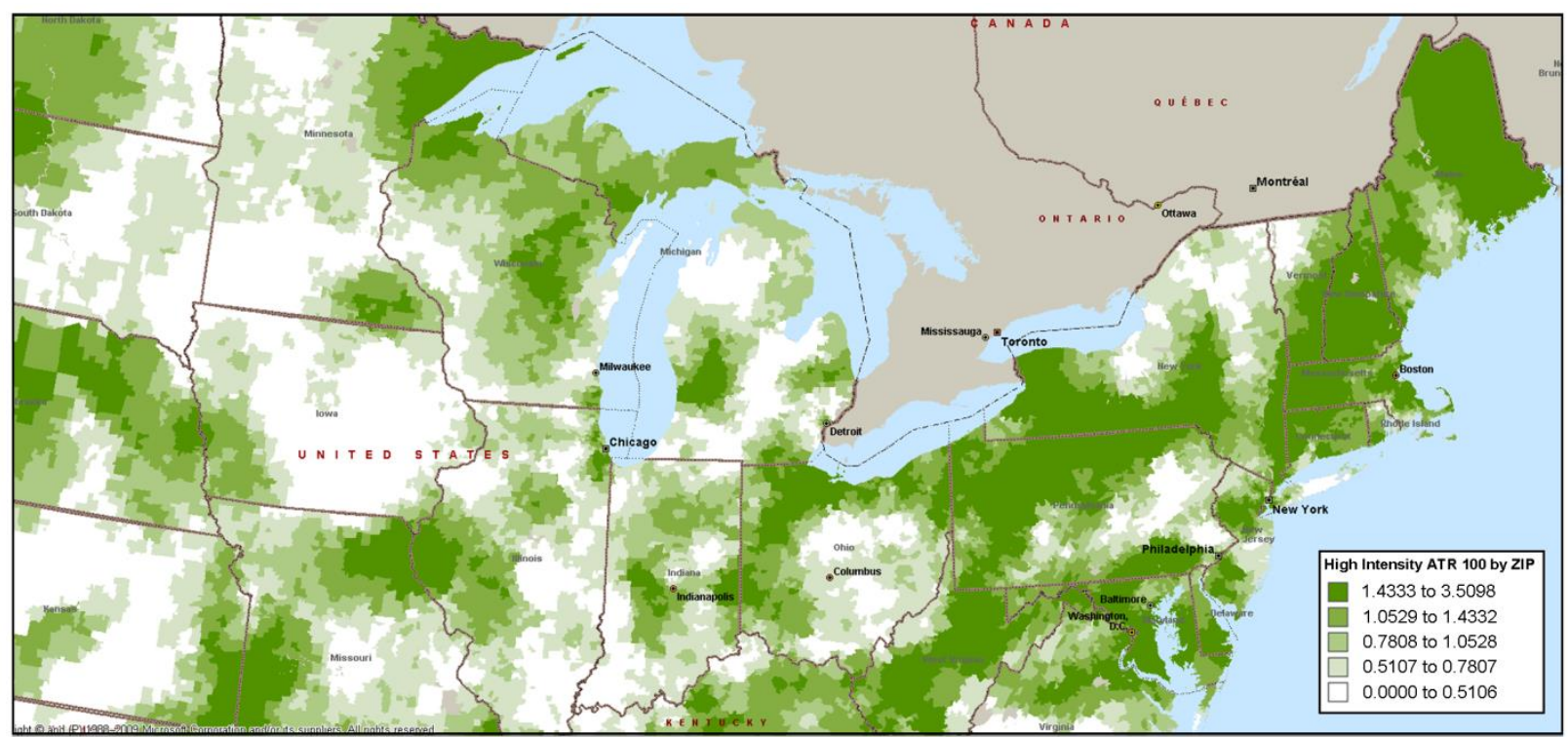


This is a final peer-reviewed manuscript.

For a published version, please go to http://journals.Iww.com/lww-

medicalcare/Abstract/2014/03001/Geographic_Variation_in_Statin_Use_for_Complex.9.aspx.

Figure 2: Northeastern United States "No Statin" Area Treatment Ratios by ZIP Code.

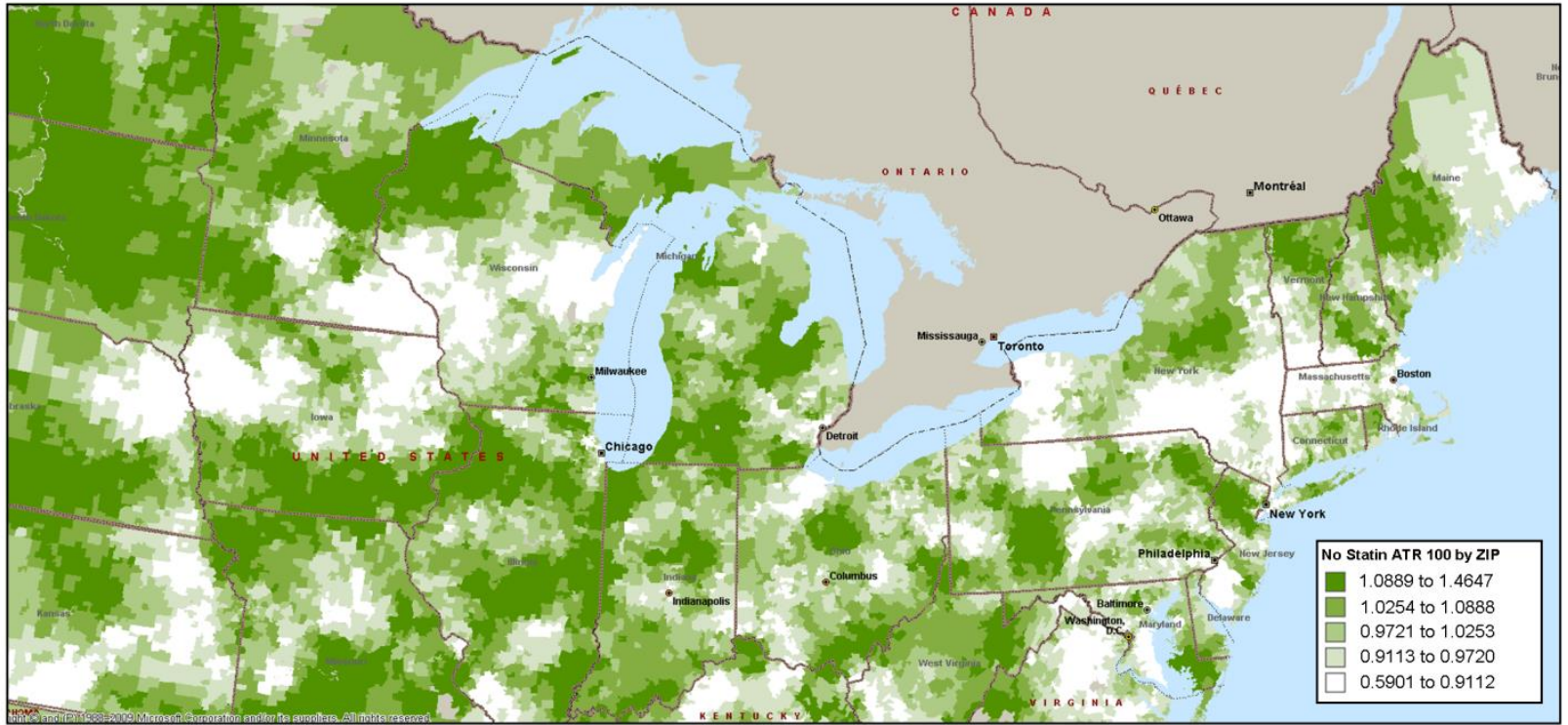


1. Wallace E, Smith SM, Fahey T. Variation in medical practice: getting the balance right. Family Practice. 2012;29(5):501-502.

2. National Institute for Health and Clinical Excellence (NICE). Statins for the Prevention of Cardiovascular Events. 2006 [updated 2008]. http://www.nice.org.uk/nicemedia/pdf/TA094guidance.pdf. Accessed March 1, 2013.

3. Dartmouth Atlas of Health Care. Effective Care. 2013; http://www.dartmouthatlas.org/keyissues/issue.aspx?con=2939. Accessed March 1, 2013.

4. Fisher ES, Wennberg JE. Health Care Quality, Geographic Variations, and the Challenge of Supply-Sensitive Care. Perspectives in Biology and Medicine. 2003;46(1):69-79.

5. Wennberg JE, Fisher ES, Skinner J. Geography and the Debate Over Medicare Reform. Health Affairs. 2002. http://www.healthaffairs.org/WebExclusives/Wennberg Web Excl 021302.htm.

6. Grundy SM, Cleeman JI, Merz CNB, et al. Implications of recent clinical trials for the National Cholesterol Education Program Adult Treatment Panel III guidelines. Circulation. 2004;110:227-239.

7. Smith SC, Jr., Allen J, Blair SN, et al. AHA/ACC guidelines for secondary prevention for patients with coronary and other atherosclerotic vascular disease: 2006 update: Endorsed by the National Heart, Lung, and Blood Institute. Circulation. 2006;113:2363-2372.

8. Armitage J, Bowman L, Wallendszus $\mathrm{K}$, et al. Intensive lowering of LDL cholesterol with $80 \mathrm{mg}$ versus $20 \mathrm{mg}$ simvastatin daily in 12,064 survivors of myocardial infarction: a double-blind randomised trial. Lancet. 2010;376(9753):1658-1669.

9. Baigent C, Blackwell L, Emberson J, et al. Efficacy and safety of more intensive lowering of LDL cholesterol: a meta-analysis of data from 170,000 participants in 26 randomised trials. Lancet. 2010;376(9753):1670-1681.

10. Law MR, Wald NJ, Rudnicka AR. Quantifying effect of statins on low density lipoprotein cholesterol, ischaemic heart disease, and stroke: systematic review and meta-analysis. British Medical Journal. 2003;326(7404):1423-1427.

11. Robinson J, Davidson M. Combination therapy with ezetimibe and simvastatin to acheive aggressive LDL reduction. Expert Rev Cardiovasc Ther. 2006;4:461476.

12. Cannon CP, Steinberg BA, Murphy SA, Mega JL, Braunwald E. Meta-analysis of cardiovascular outcomes trials comparing intensive versus moderate statin therapy. J Am Coll Cardiol. 2006;48:438-445.

13. Mills EJ, O'Regan C, Eyawo O, et al. Intensive statin therapy compared with moderate dosing for prevention of cardiovascular events: a meta-analysis of $>40$ 000 patients. European Heart Journal. 2011;32(11):1409-U1138.

14. Meek CL, Reston JD, Ramsbottom T, Pathmanathan H, Viljoen A. Use of highintensity statin therapy with simvastatin $80 \mathrm{mg}$ and atorvastatin $80 \mathrm{mg}$ in primary care. Int J Clin Pract. 2011;65(2):120-126.

15. Crouse III JR, Elam MB, Robinson JG, Ambrosius WT, Ginsberg HN. Cholesterol management: Targeting a lower low-density lipoprotein cholesterol concentration 
increases Adult Treatment Panel-III goal attainment. Am J Cardiol. 2006;97:1667-1669.

16. Javed U, Deedwania PC, Bhatt DL, et al. Use of intensive lipid-lowering therapy in patients hospitalized with acute coronary syndrome: an analysis of 65,396 hospitalizations from 344 hospitals participating in Get With The Guidelines (GWTG). Am Heart J. 2010;160(6):1130-1136, 1136 e1131-1133.

17. Lewis WR, Ellrodt G, Peterson E, et al. Trends in the Use of Evidence-Based Treatments for Coronary Artery Disease Among Women and the Elderly: Findings From the Get With the Guidelines Quality-Improvement Program. Circulation. 2009;2:633-641.

18. McGinnis BD, Olson KL, Delate TM, Stolcpart RS. Statin adherence and mortality in patients enrolled in a secondary prevention program. Am J Manag Care. 2009;15(10):689-695.

19. Zuckerman IH, Yin X, Rattinger GB, et al. Effect of exposure to evidence-based pharmacotherapy on outcomes after acute myocardial infarction in older adults. $J$ Am Geriatr Soc. 2012;60(10):1854-1861.

20. Lee HY, Cooke CE, Robertson TA. Use of Secondary Prevention Drug Therapy in Patients With Acute Coronary Syndrome After Hospital Discharge. Journal of Managed Care Pharmacy. 2008;14(3):271-280.

21. Donohue JM, Morden NE, Gellad WF, et al. Sources of Regional Variation in Medicare Part D Drug Spending. New England Journal of Medicine. 2012;366(6):530-538.

22. Yan AT, Yan RT, Tan M, et al. Contemporary management of dyslipidemia in high-risk patients: Targets still not met. Am J Med. 2006;119:676-683.

23. Robinson JG, Stone NJ. Identifying patients for aggressive cholesterol lowering: The risk curve concept. Am J Cardiol. 2006;98:1405-1408.

24. Heart Protection Study Collaborative G. MRC/BHF Heart Protection Study of cholesterol lowering with simvastatin in 5963 people with diabetes: a randomised placebo-controlled trial. Lancet. 2003;361:2005 - 2016.

25. Shepherd J, Kastelein JJP, Bittner V, et al. Intensive lipid lowering with atorvastatin in patients with coronary heart disease and chronic kidney disease: The TNT (Treating to New Targets) study. J Am Coll Cardiol. 2008;51:14481454.

26. Kjekshus J, Apetrei E, Barrios V, et al. Rosuvastatin in older patients with systolic heart failure. N Engl J Med. 2007;357:2248-2261.

27. Gissi-HF investigators. Effect of rosuvastatin in patients with chronic heart failure (the GISSI-HF trial): a randomised, double-blind, placebo-controlled trial. Lancet. 2008;372:1231-1239

28. Desai DA, Zakaria S, Ouyang P. Initiation of statin therapy: are there age limits? Curr Atheroscler Rep. 2012;14(1):17-25.

29. Thompson PD, Clarkson P, Karas RH. Statin-associated myopathy. JAMA. 2003;289(13):1681-1690.

30. Waters DD. Safety of high-dose atorvastatin therapy. Am J Cardiol. 2005;96(5A):69F-75F. 
31. Bruckert E, Hayem G, Dejager S, Yau C, Begaud B. Mild to moderate muscular symptoms with high-dosage statin therapy in hyperlipidemic patients - The PRIMO study. Cardiovascular Drugs and Therapy. 2005;19(6):403-414.

32. Abd TT, Jacobson TA. Statin-induced myopathy: a review and update. Expert Opin Drug Saf. 2011;10(3):373-387.

33. Nichols GA, Koro CE. Does statin therapy initiation increase the risk for myopathy? An observational study of 32,225 diabetic and nondiabetic patients. Clinical Therapeutics. 2007;29(8):1761-1770.

34. Golomb BA, McGraw JJ, Evans MA, Dimsdale JE. Physician response to patient reports of adverse drug effects: implications for patient-targeted adverse effect surveillance. Drug Saf. 2007;30(8):669-675.

35. Golomb BA, Evans MA. Statin adverse effects : a review of the literature and evidence for a mitochondrial mechanism. Am J Cardiovasc Drugs. 2008;8(6):373-418.

36. Florentin M, Elisaf MS. Simvastatin interactions with other drugs. Expert Opin. Drug Saf. 2012;11(3):439-444.

37. Sathasivam S. Statin induced myotoxicity. Eur J Intern Med. 2012;23(4):317-324.

38. Hedenmalm K, Alvan G, Ohagen P, Dahl ML. Muscle toxicity with statins. Pharmacoepidemiol Drug Saf. 2010;19(3):223-231.

39. Holbrook A, Wright M, Sung M, Ribic C, Baker S. Statin-associated rhabdomyolysis: is there a dose-response relationship? Can J Cardiol. 2011;27(2):146-151.

40. Armitage J. The safety of statins in clinical practice. Lancet. 2007;370(9601):1781-1790.

41. Cziraky MJ, Willey VJ, McKenney JM, et al. Statin safety: An assessment using an administrative claims database. American Journal of Cardiology. 2006;97(8A):61C-68C.

42. Ickowicz E, Panel AGSE. Patient-Centered Care for Older Adults with Multiple Chronic Conditions: A Stepwise Approach from the American Geriatrics Society. J. Am. Geriatr. Soc. 2012;60(10):1957-1968.

43. Nallamothu BK, Hayward RA, Bates ER. Beyond the Randomized Controlled Trial: The Role of Effectiveness Studies in Evaluating Cardiovascular Therapies. Circulation. 2008;118:1294-1303.

44. Zulman DM, Sussman JB, Chen X, Cigolle CT, Blaum CS, Hayward RA. Examining the evidence: a systematic review of the inclusion and analysis of older adults in randomized controlled trials. J Gen Intern Med. 2011;26(7):783790.

45. Cook EA, Schneider KM, Chrischilles EA, Brooks JM. Accounting for Immeasurable Time Bias when using Medicare Prescription Drug Data. Medicare and Medicaid Research Review. 2013;draft; under review.

46. Fang G, Brooks JM, Chrischilles EA. A New Method to Measure Geographic Variation in Prescription Use and Its Implications for Comparative Effectiveness Research. Medical Care. 2010;40:710-717.

47. Fang G, Brooks JM, Chrischilles EA. Comparison of Instrumental Variable Analysis Using a New Instrument With Risk Adjustment Methods to Reduce 
This is a final peer-reviewed manuscript.

For a published version, please go to http://journals.Iww.com/lww-

medicalcare/Abstract/2014/03001/Geographic_Variation_in_Statin_Use_for_Complex.9.aspx.

Confounding by Indication. American Journal of Epidemiology.

2012;175(11):1142-1151.

48. Brooks JM, Chrischilles EA, Landrum MB, et al. Survival implications associated with variation in mastectomy rates for early-staged breast cancer. Int J Surg Oncol. 2012;2012:127854.

49. Armitage P. Tests for Linear Trends in Proportions and Frequencies. Biometrics. 1955;11:375-386.

50. Wennberg JE. Which Rate is Right? New England Journal of Medicine. 1986;315(13):810-815. 